\title{
El módulo espacial como elemento de acondicionamiento ambiental: el pabellón de España de Corrales y Molezún
}

\section{The spatial module as environmental conditioning element: the Spanish pavilion by Corrales and Molezun}

\author{
$\underline{\text { R. Suárez }}^{(*)}$, G. Pitel ${ }^{(*)}$, R. Escandón ${ }^{(*)}$
}

RESUMEN

En los años 50 surge una revisión del Movimiento Moderno, que incorpora la seriación modular y la conexión con el contexto ambiental, aunque con unas premisas alejadas de los actuales paradigmas de sostenibilidad. En este contexto, dentro del ámbito nacional, destaca el pabellón de España de la Exposición Universal de Bruselas de 1958, de Corrales y Molezún. Este trabajo pretende desvelar cuantitativamente el comportamiento ambiental del pabellón en sus dos ubicaciones y configuraciones, Bruselas y Madrid, mediante la simulación y análisis de los modelos energéticos y lumínicos que reproducen las características del pabellón, con el fin de aportar un nuevo enfoque crítico, valorando la eficiencia del módulo para adaptarse a distintas condiciones ambientales. El análisis realizado revela la influencia del clima, la compacidad y la orientación, así como la problemática del confort térmico asociado a la iluminación natural cuando los porcentajes de acristalamiento son importantes y en entornos con alta radiación solar.

Palabras clave: seriación modular; acondicionamiento ambiental; eficiencia energética; iluminación natural; confort; sistemas pasivos.

\section{ABSTRACT}

In the 50 a review of Modern Movement, which assimilates modular serialization and a connection with the environmental context, although with remote premises of the contemporary paradigms of sustainability arise. In this context, within national stage, stands out the Spanish pavilion at the Brussels International Exhibition in 1958 by Corrales and Molezún. This work seeks a quantitatively reveal of the environmental performance of the pavilion in its two locations and settings, in Brussels and Madrid, through simulation and analysis of energy and lighting models which reproduces the characteristic of the pavilion with the purpose of contributing to give a new critical point of view, valuing the module efficiency to adapt to different environmental conditions. The completed analysis reveals the influence of the climate, compactness and orientation, as in the difficulties associated with thermal comfort and natural light when glazing percentage are important and there are high solar radiation settings.

Keywords: environmental conditioning; modular serialization; energy efficiency; natural lighting; comfort; passive systems.

(*) Instituto Universitario de Arquitectura y Ciencias de la Construcción - Escuela Técnica Superior de Arquitectura, Universidad de Sevilla, Sevilla (España).

Persona de contacto/Corresponding author: rsuarez@us.es (R. Suárez)

ORCID: http://orcid.org/oooo-ooo1-6136-1596 (R. Suárez); http://orcid.org/oooo-0oo2-2162-7780 (G. Pitel);

http://orcid.org/oooo-0003-0531-698X (R. Escandón)

Cómo citar este artículo/Citation: Suárez, R., Pitel, G., Escandón, R. (2017). El módulo espacial como elemento de acondicionamiento ambiental: el pabellón de España de Corrales y Molezún. Informes de la Construcción, 69(546): e195, doi: http://dx.doi.org/10.3989/ ic.16.133.

Copyright: (C) 2017 CSIC. Licencia / License: Salvo indicación contraria, todos los contenidos de la edición electrónica de Informes de la Construcción se distribuyen bajo una licencia de uso y distribución Creative Commons Attribution License (CC BY) Spain 3.o. 


\section{INTRODUCCIÓN}

La actual sobrevaloración del término sostenibilidad y la incorporación de lo termodinámico a la arquitectura parece ser ajeno a concepciones arquitectónicas basadas en la seriación modular y la materialidad industrial, una arquitectura desarrollada principalmente en los años 50 a partir del Team X, como revisión de los dogmatismos elementales y mecanicistas del Movimiento Moderno (1) (2) que acaban en el Estilo Internacional con la mecanización del ambiente. Frente a estos postulados la nueva visión arquitectónica incorpora el acondicionamiento pasivo y el contexto ambiental, utilizando el módulo como una unidad espacial reconocible en el conjunto por su geometría, dentro de un entramado bidireccional flexible, de crecimiento extensible que genera una neutralidad espacial.

Las fuentes bibliográficas tradicionales analizan la arquitectura del Movimiento Moderno desde visiones formales, funcionales (3) o historiográficas (4), comenzando en los años 60 las primeras revisiones desde una óptica de entornos habitables, con autores como V. Olgyay (5) o R. Banham (6). El análisis de importantes obras del Movimiento Moderno desde el punto de vista ambiental es más reciente, con autores como C. Porteous (7), D. Hawkees (8) o R. Cole (9), que introducen la relación clima-arquitectura, pero desde una visión descriptiva y no cuantitativa. También cabe destacar estudios recientes de la sostenibilidad en la arquitectura modular o industrializada, enfocados fundamentalmente en la reducción de emisiones de $\mathrm{CO}_{2}$ durante el ciclo de vida de los edificios (10).

Sin embargo, recientes trabajos analizan, desde una posición técnica y científica, los sistemas de acondicionamiento ambiental propuestos por maestros del Movimiento Moderno. Destacan los trabajos sobre las aportaciones de Le Corbusier como el brise - soleil y el aerateur realizados por I. Requena (11) mediante las actuales técnicas de simulación para realizar un análisis cuantitativo de su influencia sobre las condiciones ambientales (humedad, ventilación, soleamiento, radiación y luminancia), el trabajo de C. Ramírez et al. (12) que analiza mediante modelos matemáticos el muro neutralizante y la respiración exacta como sistemas de acondicionamiento ambiental propuestos por Le Corbusier en la Ciudad del Refugio; o el análisis ambiental de la obra de los Smithsons a través de su laboratorio ambiental, el pabellón UpperLawn realizado por R. Escandón et al. (13).

El objetivo de este trabajo es valorar desde un punto de vista ambiental, con la ayuda de las actuales herramientas informáticas de simulación ambiental y energética, el pabellón de España en la Exposición Universal de Bruselas de 1958 de José Antonio Corrales y Ramón Vázquez Molezún, reconstruido en el año 1959 en la Casa de Campo de Madrid, como ejemplo relevante de arquitectura española basada en la seriación modular. Partiendo de la unidad constructiva elemental como principio generador del espacio, se analiza y valora el comportamiento ambiental y la eficiencia energética del módulo para adaptarse a diferentes condiciones ambientales, en concreto el soleamiento, la iluminación natural y la evolución térmica y energética del pabellón tanto en su emplazamiento original en Bruselas, como en su adaptación posterior en Madrid.

\section{CASO DE ESTUDIO: EL PABELLÓN DE ESPAÑA}

El pabellón de España para la Exposición Universal de Bruselas de 1958 de José Antonio Corrales y Ramón Vázquez Mo- lezún es un hito en la historia contemporánea de la arquitectura española, y puede ser considerado como la primera gran obra española de proyección internacional, adelantándose a la arquitectura de seriación modular propuesta por el Team X.

El pabellón parte de la seriación modular mediante una unidad constructiva elemental: una cubierta hexagonal prefabricada y ligera de seis metros de diámetro con un apoyo central, que facilita una mayor flexibilidad y libertad al establecer tres direcciones de acción, generando una arquitectura orgánica basada en la abstracción compositiva. Mediante un sistema de composición flexible, basado en diferentes alturas del módulo, se generan unos lucernarios, que junto con diferentes grados de opacidad del cerramiento son los encargados de iluminar naturalmente el espacio interior (Figuras 1 y 2).

«El espacio infinito, discontinuo y diverso del Pabellón de Bruselas, capaz de adaptarse a cualesquiera que sean las condiciones de perímetro y topografía, es el feliz desenlace a que lleva la consecuente y precisa manipulación de un elemento modular que es a un tiempo capaz de estructurar y de erosionar el volumen que resulta» (14).

Su traslado a Madrid en 1969, tras la finalización de la Exposición Universal, demuestra su capacidad de reversibilidad, adaptabilidad y manipulación. Frente a la posibilidad de reconstrucción, Corrales y Molezún optaron por utilizar la imagen del pabellón en un entorno y un clima diferentes. Organizan la planta del pabellón alrededor de las encinas existentes, creando tres patios interiores, cerrándose al exterior y abriéndose a los patios (Figura 1). El resultado es un nuevo edificio que mantiene la idea original, ya que su propósito en Madrid fue reconstruir una idea, un concepto como expresan los autores:

«... lo bonito de este pabellón es que es tan flexible... tengo tantas piezas y tengo que utilizarlas, puedes montarlas de mil maneras...» (15).

Con el cese de las Ferias del Campo en 1975, el pabellón vuelve a caer en desuso y se deteriora muy rápidamente. Su actual abandono, en un lamentable estado de conservación, prácticamente en ruina, es una razón más para intentar recuperar un edificio mítico de nuestra historia de la arquitectura del siglo XX.

\subsection{Localización y descripción del clima}

El pabellón se sitúa en dos ubicaciones con dos climas totalmente distintos.

\subsubsection{Bruselas}

El pabellón de España se encontraba localizado en el parque Heysel de Bruselas, en la latitud $50^{\circ} 85^{\prime} \mathrm{N}$, longitud $4^{\circ} 22^{\prime}$ $60 " \mathrm{E}$, y altitud $64 \mathrm{~m}$. El clima de Bruselas es marítimo temperado con inviernos relativamente suaves, algunos períodos de frío intenso, veranos templados y agradables e importantes precipitaciones repartidas a lo largo del año. Las temperaturas oscilan desde los $3{ }^{\circ} \mathrm{C}$ de media en invierno hasta máximas de $23{ }^{\circ} \mathrm{C}$ en verano (Tabla 1 ).

\subsubsection{Madrid}

En su localización definitiva el pabellón de España se encuentra localizado en la Casa de Campo de Madrid: latitud 

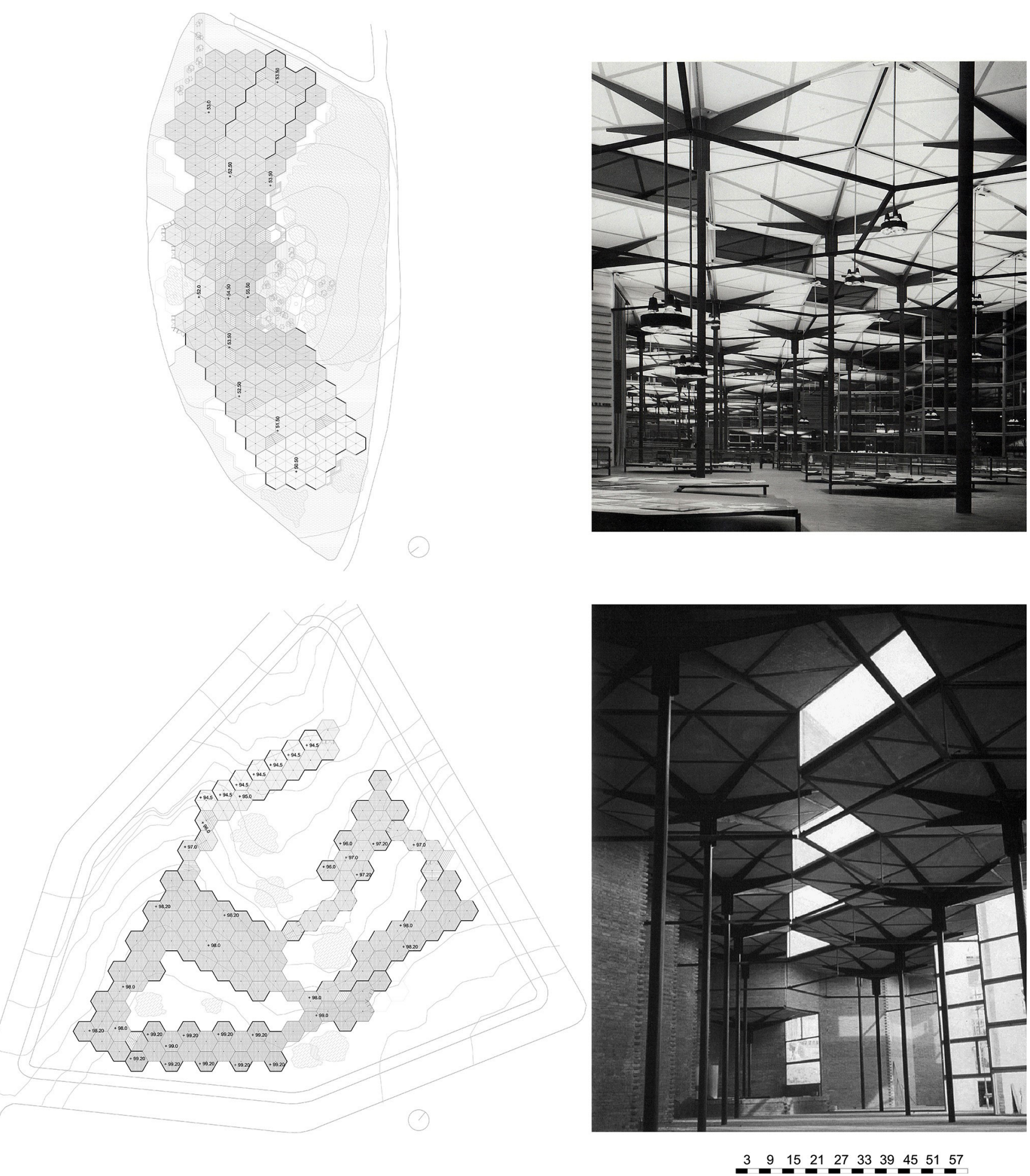

Figura 1. Planta del pabellón e imagen interior en Bruselas (superior) y en Madrid (inferior). Fuente imágenes: Cánovas, A. (Eds.) (16).

$40^{\circ} 26^{\prime} \mathrm{N}$, longitud $3^{\circ} 41^{\prime} \mathrm{O}$ y una altitud de $667 \mathrm{~m}$. El clima de Madrid es mediterráneo continental con inviernos fríos y veranos calurosos, con temperaturas medias máximas que superan los $30^{\circ} \mathrm{C}$ (Tabla 1$)$.

\subsection{Envolvente y sistemas constructivos}

El pabellón está compuesto por la yuxtaposición de un módulo hexagonal «paraguas o sombrilla» con un fuste metálico y cubierta ligera que es al mismo tiempo sistema estructural del pabellón. Estos módulos se cierran en sus límites con el exterior mediante un cerramiento de una hoja simple de medio pie de ladrillo, sin aislamiento térmico, que presenta diferentes porcentajes de huecos en función de su posición en la envolvente, con vidrio simple y carpintería de aluminio, incorporando ventanas oscilantes de eje horizontal para la ventilación. La cubierta es un elemento muy ligero de una hoja de un panel de cemento con fibras de madera, que tampoco se encuentra aislado térmicamente (Tabla 2).

En su nueva ubicación en Madrid se hizo un cambio en la envolvente con respecto al edificio en Bruselas, instalando una serie de ventanas con celosías para la ventilación manual del pabellón, para intentar paliar las altas temperaturas en verano. 

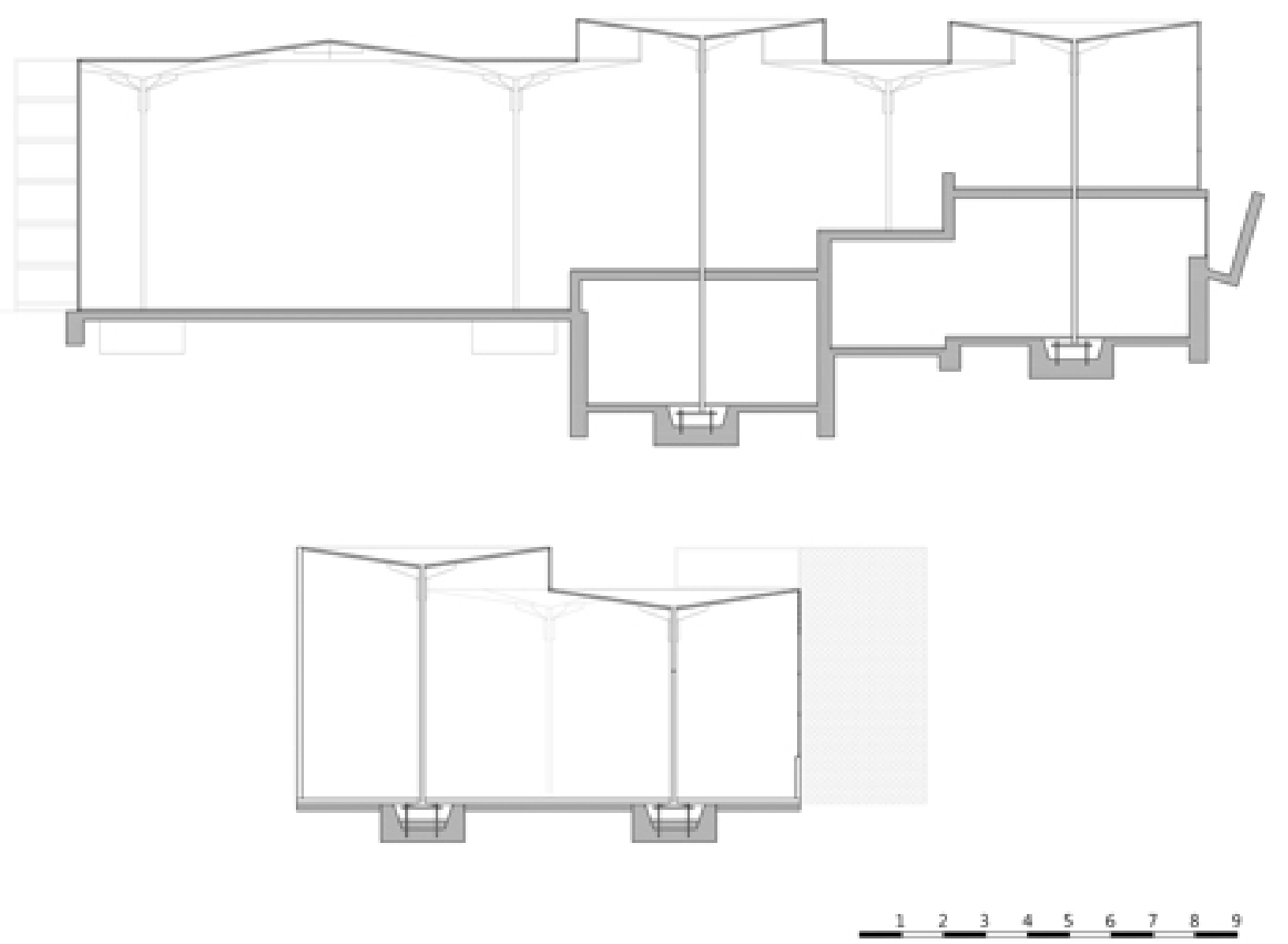

Figura 2. Secciones del pabellón: superior en Bruselas e inferior en Madrid.

Tabla 1. Datos climáticos de Bruselas (BR), Meteo Belgique (servicio meteorológico nacional de Bélgica) y de Madrid (MD), AEMET (Agencia Estatal de Meteorología).

\begin{tabular}{|c|c|c|c|c|c|c|c|c|c|c|c|c|c|c|c|c|c|c|c|c|c|c|c|c|}
\hline & \multicolumn{2}{|c|}{ ENE } & \multicolumn{2}{|c|}{ FEB } & \multicolumn{2}{|c|}{ MAR } & \multicolumn{2}{|c|}{ ABR } & \multicolumn{2}{|c|}{ MAY } & \multicolumn{2}{|c|}{ JUN } & \multicolumn{2}{|c|}{ JUL } & \multicolumn{2}{|c|}{ AGO } & \multicolumn{2}{|c|}{ SEP } & \multicolumn{2}{|c|}{ OCT } & \multicolumn{2}{|c|}{ NOV } & \multicolumn{2}{|c|}{ DIC } \\
\hline & BR & MD & BR & MD & BR & MD & BR & MD & BR & MD & BR & MD & BR & MD & BR & MD & BR & MD & BR & MD & BR & MD & BR & MD \\
\hline $\begin{array}{l}\text { Temperatura } \\
\text { media máxima } \\
\left({ }^{\circ} \mathrm{C}\right)\end{array}$ & 5,7 & 11 & 6,6 & 13 & 10 & 17 & 14,2 & 18,7 & 18,1 & 23,1 & 20,6 & 29,5 & 23 & 33,5 & 22,6 & 32,8 & 19 & 27,2 & 15 & 21 & 9,5 & 15 & 6,1 & 10,9 \\
\hline $\begin{array}{l}\text { Temperatura } \\
\text { media mínima } \\
\left({ }^{\circ} \mathrm{C}\right)\end{array}$ & 0,7 & 0,2 & 0,7 & 1,2 & 3,1 & 3,5 & 5,3 & 5,7 & 9,2 & 9,3 & 11,9 & 13,9 & 14 & 16,8 & 13,6 & 16,5 & 10,9 & 13,1 & 7,8 & 8,7 & 4,1 & 4,1 & 1,6 & 1,4 \\
\hline $\begin{array}{l}\text { Media de días } \\
\text { con heladas }\end{array}$ & 5,2 & 16 & 5,9 & 12 & 3,2 & 4,7 & 2,4 & 0,9 & 0,4 & 0,1 & 0 & 0 & 0 & 0 & 0 & 0 & 0 & 0 & 0 & 0,2 & 2,4 & 5,4 & 4,6 & 12,8 \\
\hline $\begin{array}{l}\text { Media de horas } \\
\text { sol }\end{array}$ & 59 & 144 & 77 & 168 & 114 & 224 & 159 & 226 & 191 & 258 & 188 & 310 & 201 & 354 & 190 & 329 & 143 & 258 & 113 & 199 & 66 & 151 & 45 & 128 \\
\hline $\begin{array}{l}\text { Media de lluvia } \\
(\mathrm{mm})\end{array}$ & 76,1 & 29 & 63,1 & 32 & 70 & 22 & 51,3 & 38 & 66,5 & 44 & 71,8 & 22 & 3,5 & 9 & 79,3 & 10 & 68,9 & 24 & 75 & 51 & 46,4 & 49 & 81 & 42 \\
\hline $\begin{array}{l}\text { Media de días } \\
\text { lluvia > } 1 \mathrm{~mm}\end{array}$ & 19,2 & 5,2 & 16,6 & 4,7 & 18 & 3,5 & 15,9 & 6,4 & 16,2 & 6,8 & 15 & 3,6 & 14,3 & 1,5 & 14,5 & 1,5 & 15,7 & 2,9 & 17 & 6,6 & 18,8 & 6,2 & 19,3 & 6,3 \\
\hline
\end{tabular}

\subsection{Estrategias de acondicionamiento pasivo}

Desde el punto de vista ambiental las estrategias de acondicionamiento pasivo del pabellón en Bruselas se centraron principalmente en el tratamiento de la luz natural, mediante los lucernarios y el acristalamiento de la fachada, mientras que cuando el pabellón se reconstruye en Madrid se complementa la iluminación natural con unas estrategias de control térmico ya que «siendo la exposición en primavera y verano, es aconsejable procurar cerrar huecos a poniente y mediodía debido, a la rigurosa temperatura estival en Madrid» (18) (Figura 3).

\subsection{Sistemas de acondicionamiento térmico}

En su ubicación en Bruselas el pabellón de España contaba con dos sistemas de acondicionamiento situados en el sótano: una red de aire caliente canalizado con impulsión en los frentes de las bancadas que se calentaba con dos calderas, y un sistema de apoyo mediante unos tréboles con tubos infrarrojos en la parte alta de la estructura, bajo el techo. Aunque ambas instalaciones se construyeron, no hay constancia de que se llegaran a utilizar durante la exposición de Bruselas, dado que ésta se desarrolló durante primavera y verano. Tras su traslado a Madrid se eliminan los sistemas previstos de 
Tabla 2. Caracterización constructiva y térmica de la envolvente.

\begin{tabular}{|c|c|c|c|c|c|}
\hline \multirow{2}{*}{ Cerramiento } & \multirow{2}{*}{ Descripción } & \multirow{2}{*}{$\mathbf{U} \mathbf{W} / \mathbf{m}^{2} \mathbf{K}$} & \multicolumn{2}{|c|}{ Superficie $\left(\mathrm{m}^{2}\right)$} & \multirow{2}{*}{ Orientación } \\
\hline & & & Bruselas & Madrid & \\
\hline \multirow{4}{*}{ Fachada } & \multirow{4}{*}{$1 / 2$ pie de ladrillo perforado } & \multirow{4}{*}{2,6} & 180 & 480 & $\mathrm{~N}$ \\
\hline & & & 282 & 468 & $\mathrm{~S}$ \\
\hline & & & $\mathrm{o}$ & 471 & $\mathrm{E}$ \\
\hline & & & 183 & 1.077 & $\mathrm{O}$ \\
\hline Cubierta & $\begin{array}{l}\text { Lámina aluminio, pieza de cemento durisol } \\
\text { y fibras de madera y capa de pintura }\end{array}$ & 2,4 & 3.112 & 2.853 & \\
\hline \multirow{4}{*}{ Hueco fachada } & \multirow{4}{*}{$\begin{array}{l}\text { Vidrio simple } 6 \mathrm{~mm} \text {. } \\
\text { Marco de aluminio }\end{array}$} & \multirow{4}{*}{$\begin{array}{l}5,7 \\
5,7\end{array}$} & 306 & 267 & $\mathrm{~N}$ \\
\hline & & & 204 & 225 & $\mathrm{~S}$ \\
\hline & & & 354 & 333 & $\mathrm{E}$ \\
\hline & & & 411 & 267 & $\mathrm{O}$ \\
\hline \multirow{4}{*}{ Lucernario } & \multirow{4}{*}{$\begin{array}{l}\text { Vidrio simple } 6 \mathrm{~mm} \text {. } \\
\text { Marco de aluminio }\end{array}$} & \multirow{4}{*}{$\begin{array}{l}5,7 \\
5,7\end{array}$} & 30 & 33 & $\mathrm{~N}$ \\
\hline & & & 21 & 69 & $\mathrm{~S}$ \\
\hline & & & 60 & 132 & $\mathrm{E}$ \\
\hline & & & 153 & 83 & $\mathrm{O}$ \\
\hline Suelo & Solera con revestimiento cerámico & $\begin{array}{c}\text { 0,27 Bruselas } \\
\text { 0,47 Madrid }\end{array}$ & 3.112 & 2.853 & \\
\hline
\end{tabular}

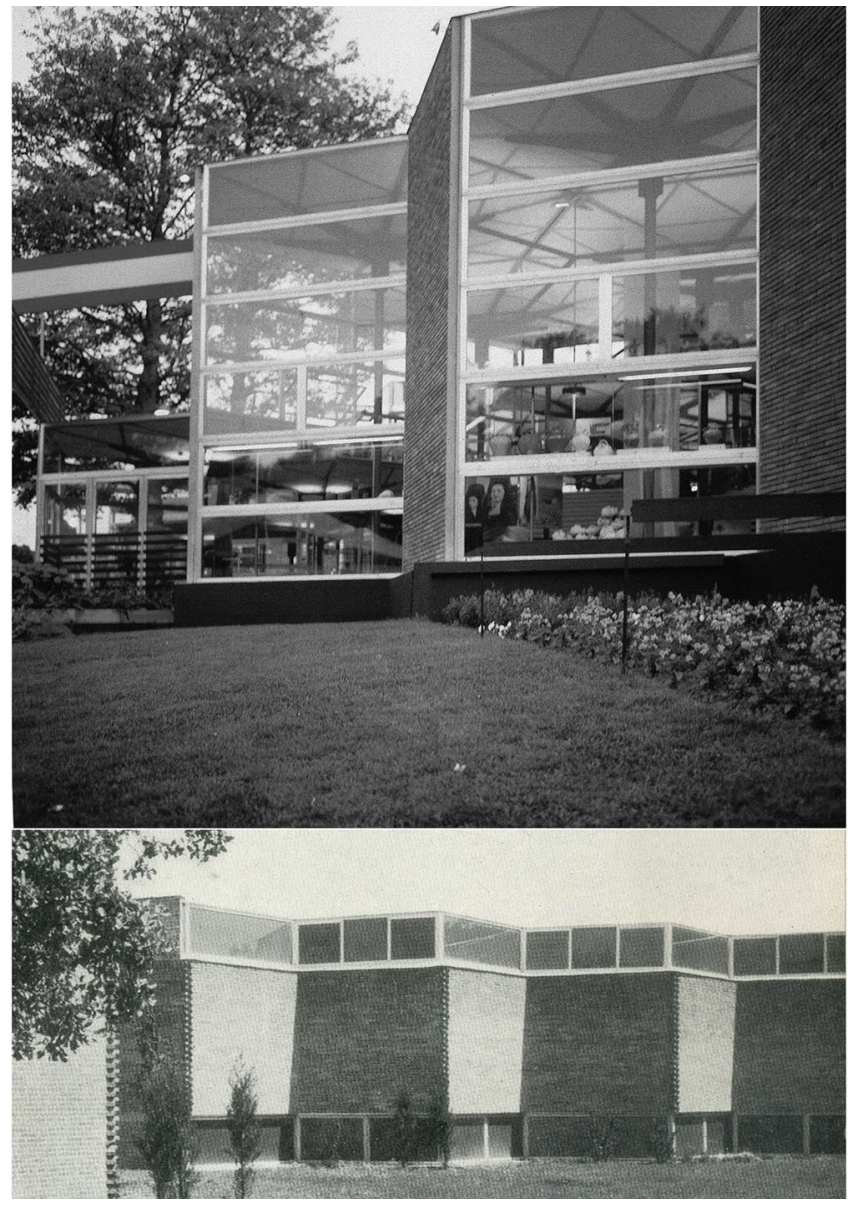

Figura 3. Fachadas del pabellón: superior en Bruselas (Fuente: Cánovas, A., Eds.) (16) e inferior en Madrid (Fuente: Fernández del Amo) (17).

calefacción en Bruselas y no se diseñó ningún tipo de instalación de climatización ni de ventilación mecánica.

\section{DESCRIPCIÓN DE LA METODOLOGÍA}

En este estudio del pabellón de España se ha aplicado una metodología que nos permite valorar cuantitativamente el acondicionamiento ambiental del pabellón para conocer sus estrategias de acondicionamiento pasivo y su adaptación a dos climas diferentes evaluando, principalmente, las condiciones de iluminación natural y el comportamiento térmico del pabellón.

A la hora de realizar un análisis térmico y de iluminación natural surge el problema de la integración del análisis. Si bien en los últimos años han surgido diferentes propuestas para afrontar este análisis de una manera integral mediante EnergyPlus (19), esta opción es compleja en su funcionamiento y necesita largos tiempos de procesado. Otra opción es recurrir a herramientas más simples, que intentan integrar un módulo de iluminación en un programa de análisis térmico (20), importando las características térmicas y lumínicas desde un programa WIS (Window Information System) (21), pero, sin embargo, es difícil obtener resultados satisfactorios de forma integrada. Por ello se plantea una metodología que permita la evaluación térmica y lumínica mediante un mismo modelo de forma dinámica, cuantificando su evolución mensual, diaria y horaria, evaluado mediante distintas herramientas de simulación:

El estudio de soleamiento mediante el programa Autodesk Ecotect Analysis (22). Permite visualizar de forma sencilla la incidencia solar y la radiación en los cerramientos, además de mostrar la posición solar y el recorrido relativo con respecto al modelo, en cualquier fecha, hora y ubicación.

- El estudio de la iluminación natural mediante el programa Daylight Visualizer de Velux (23) que permite simular y cuantificar la iluminación natural en el interior del pabellón, tanto estática como dinámica a lo largo del año, para distintos tipos de cielo. La precisión del programa ha sido validada con su contraste con los cálculos analíticos del algoritmo de Tregeza y los test de CIE (24).

- El estudio energético mediante el programa DesignBuilder (25) permite obtener las demandas energéticas anuales, mensuales y diarias, así como valores horarios de temperatura en el interior del pabellón. Es una interfaz 3D que utiliza el motor de simulación térmica EnergyPlus, programa de simulación energética de edificios del U. S. DOE (Departamento de Energía de EE. UU.). 
- El actual estado de ruina del pabellón en la Casa de Campo de Madrid ha imposibilitado la realización de mediciones in situ para conocer sus condiciones ambientales interiores.

\subsection{Modelos de simulación}

El excesivo tamaño del pabellón, que conlleva unos tiempos de computación demasiados largos, junto con el carácter seriado del edificio, generado a partir de un módulo base hexagonal, aconsejan construir los modelos de simulación mediante un conjunto múltiple de módulos hexagonales que sea representativo del comportamiento general del edificio. Para la elección del modelo de simulación se ha llevado a cabo un análisis del pabellón en busca de un patrón que permita extraer un modelo para el estudio del mismo en cada una de sus ubicaciones.

En la configuración del pabellón en Bruselas existen dos patrones básicos de agrupación condicionados por la adaptación al terreno y la disposición de los lucernarios. Un primer modelo (B.1), con orientación este-oeste, en el que el plano del suelo se escalona con descensos de $1 \mathrm{~m}$ por cada módulo espacial, mientras que el plano de cubierta mantiene una configuración constante de módulo alto-módulo bajo (Figura 4.a). Un segundo modelo (B.2), con orientación norte-sur, caracterizado por tener módulos emparejados con una relación lineal del plano de suelo que se escalona con ascensos de $1 \mathrm{~m}$ cada dos módulos, y una cubierta que se adapta a la misma configuración de escalonamiento que el plano del suelo (Figura 4.b). En ambos casos los modelos presentan un único elemento de fachada mientras que el resto del conjunto es zona interior del pabellón.

En la configuración del pabellón en Madrid (M), a diferencia de Bruselas, se observa que la mayor parte del pabellón se encuentra formada por parejas de módulos, que cuentan con cerramientos en contacto con el exterior, siendo siempre un cerramiento opaco y otro transparente, con un módulo eleva-
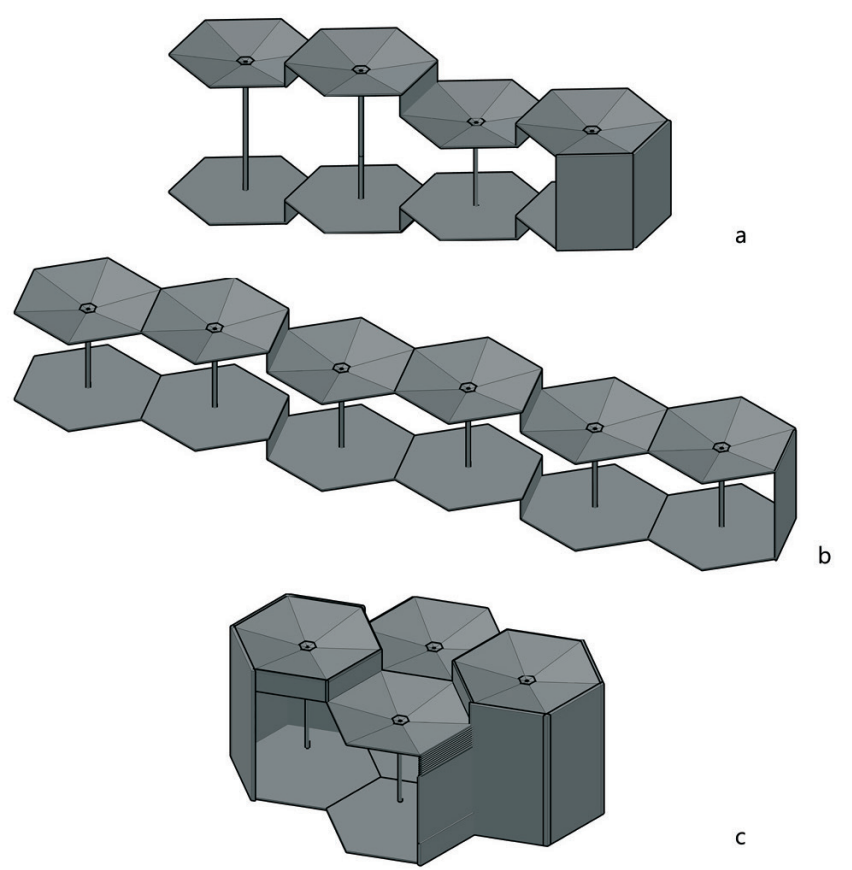

c

Figura 4. a) Modelo 1 de Bruselas (B1), b) Modelo 2 de Bruselas (B.2) y c) Modelo de Madrid (M). do, que permite una iluminación cenital (Figura 4.c). Como la distribución del módulo en planta tiene varias orientaciones, se realiza el estudio en las orientaciones principales: nortesur y este-oeste.

En estos modelos se presenta un problema de incertidumbre en las adyacencias, en las superficies virtuales de contacto con otro módulo, que provocarían ciertas desviaciones en los resultados de las simulaciones, por lo que se completa en cada modelo el módulo base con módulos a su alrededor, de manera que éstos simulen el entorno cercano, extrayéndose para su análisis los resultados del módulo base central.

\subsection{Estudio del soleamiento}

Se ha llevado a cabo un estudio del soleamiento del pabellón con la ayuda del programa Autodesk Ecotect Analysis. No se han tenido en cuenta el entorno, para valorar de forma individual el módulo, ni se han tenido en cuenta la composición de la envolvente y las cargas internas, puesto que no influyen en el estudio de soleamiento y sombras arrojadas.

Se analizan las horas en las que existe incidencia directa de luz natural en el interior del pabellón, el soleamiento sobre los elementos principales que acumulan calor y las sombras arrojadas. Sin embargo, para cuantificar las ganancias solares por superficie acristalada y comprobar si éstas pueden compensar las pérdidas térmicas durante la noche, es necesario complementar este estudio solar con un análisis ambiental y energético mediante la herramienta informática DesignBuilder.

\section{3•3. Simulación de iluminación natural}

Para el estudio de la iluminación natural con el programa Daylight Visualizer los modelos construidos no tienen en consideración los elementos del entorno, ni la composición de la envolvente, ni las cargas internas. Se han considerado dos condiciones de cielo: nublado y despejado, así como la orientación del pabellón, y los valores internos de reflectancia de los materiales que componen el modelo (Tabla 3), valorándose los siguientes indicadores:

- Estudio estático, mediante el Factor de Iluminación Natural (FIN) (26), que analiza la luz en términos relativos y permite determinar el rendimiento de luz natural de un espacio interior. Sin embargo, este estudio supone una visión limitada del comportamiento de la luz natural al no considerar la iluminancia directa del sol, por lo que no se puede considerar como un indicador de un adecuado diseño de luz natural (27).

- Estudio dinámico, considerando la cantidad y el carácter de las variaciones diarias y estacionales de luz del día, que permite analizar las condiciones anuales de iluminación natural (27) (28). Se realiza mediante la valoración de iluminación máxima, mínima y media a lo largo del año en

Tabla 3. Valor de reflectancia de los paramentos interiores.

\begin{tabular}{|l|c|}
\hline \multicolumn{1}{|c|}{ Paramento } & Valor reflectancia \\
\hline Techo & 0,84 \\
\hline Suelo & 0,17 \\
\hline Cerramiento vertical opaco & 0,5 \\
\hline Cerramiento vertical transparente & 0,68 \\
\hline Cerramiento vertical virtual & 0 \\
\hline
\end{tabular}


los equinoccios y solsticios, junto con dos indicadores: el Daylight Autonomy (DA) (29), que indica el porcentaje de horas en los que el nivel de iluminación puede ser mantenido por iluminación natural únicamente, y Useful Daylight Illuminances (UDI) (30) que indica cuándo los niveles de iluminación natural se encuentran en unos determinados rangos de utilidad, como indicador de los requerimientos visuales, estableciendo valores que no sean demasiado bajos (UDI ${ }_{<100}$ ) o demasiado altos (> 2.00o lux) que puede conllevar a incomodidad visual-térmica para el usuario. Para su análisis se ha considerado un mallado de $1,0 \times 1,0$ m en el plano del suelo.

\subsection{Simulación ambiental y energética}

La simulación ambiental y energética se ha realizado con el programa DesignBuilder, que a partir de la base de datos de EnergyPlus permite obtener datos precisos sobre temperatura interior y demandas energéticas anuales, mensuales y horarias. Para valorar las soluciones pasivas del pabellón se realiza un análisis de la evolución horaria de la temperatura exterior y operativa interior en libre evolución, durante el día representativo considerado en el análisis de iluminación natural: equinoccios y solsticios, y se relaciona con los valores de radiación solar durante dicho día. Se ha estimado una tasa constante de infiltraciones de 0,5 renovaciones/hora debida a las carpinterías originales poco estancas.

Se completa el análisis de evolución de temperaturas operativas interiores con la comparación de éstas con una banda de temperatura operativa de confort adaptativo, evaluadas a partir de los índices adaptativos de ASHRAE (31), según la ecuación:

$$
\mathrm{T}_{\mathrm{co}}=0,31 \cdot \mathrm{T}_{\mathrm{ext}, \mathrm{ref}}+17,8
$$

Donde $\mathrm{T}_{\mathrm{co}}$ es la temperatura operativa de confort, $\mathrm{y}_{\mathrm{ext}}$, ref es la temperatura exterior de referencia, considerada como media mensual. Se considera un rango de aceptación correspondiente al 90\% de satisfacción de los ocupantes, lo que supone la consideración de un intervalo de $\pm 2,5^{\circ} \mathrm{C}$ sobre el valor de cálculo.

La tasa de ventilación natural en el pabellón es un parámetro difícil de conocer, por lo que supone una incertidumbre en el análisis. Por ello se plantea el análisis del comportamiento ambiental y energético para varias tasas de ventilación: 0,5, 1 y 2 renovaciones/hora.

Para la realización de plantillas de uso, ocupación e iluminación artificial hay que tener en cuenta que el pabellón tenía en ambas ubicaciones un uso durante el período de primavera y verano, mientras que en invierno estaba previsto que permaneciera cerrado, por lo que el análisis se realiza en este período temporal.

En los modelos de simulación se han incluido como cargas internas las cargas de ocupación e iluminación, considerando las programaciones, a falta de tener más datos sobre los horarios de uso, de los perfiles de uso no residencial: $12 \mathrm{~h}$, intensidad media del apéndice C del CTE-DB HE-1, a partir de los siguientes datos:

- Número de ocupantes: 0,5 persona/m² (según CTE-DB SI-3 para espacios expositivos).

- Tasa metabólica: o,63.
Tabla 4. Programación de iluminación asociada al uso del pabellón.

\begin{tabular}{|c|c|c|c|}
\hline & $\begin{array}{c}\% \text { acristalamiento } \\
\text { fachada }\end{array}$ & Marzo & Junio \\
\hline Bruselas & о у 100 & $\begin{array}{l}\text { 07:00-14:00 } \\
\text { 17:00-20:00 }\end{array}$ & $\begin{array}{l}\text { 07:00-09:00 } \\
\text { 17:00-20:00 }\end{array}$ \\
\hline \multirow{4}{*}{ Madrid } & o & $\begin{array}{l}\text { 07:00-14:00 } \\
\text { 17:00-20:00 }\end{array}$ & $\begin{array}{l}\text { 07:00-09:00 } \\
\text { 17:00-20:00 }\end{array}$ \\
\hline & 40 & $\begin{array}{l}\text { 07:00-09:00 } \\
\text { 17:00-20:00 }\end{array}$ & $\begin{array}{l}\text { 07:00-08:00 } \\
\text { 17:00-20:00 }\end{array}$ \\
\hline & 60 & $\begin{array}{l}\text { 07:00-08:00 } \\
\text { 17:00-20:00 }\end{array}$ & 17:00-20:00 \\
\hline & 100 & $\begin{array}{l}\text { 07:00-08:00 } \\
\text { 17:00-20:00 }\end{array}$ & 18:00-20:00 \\
\hline
\end{tabular}

- Carga térmica de iluminación por superficie: para el modelo en Bruselas se ha considerado de $2,8 \mathrm{~W} / \mathrm{m}^{2}$, mientras que para el modelo en Madrid es de 6,08. Esta carga se ha obtenido a partir del sistema de iluminación proyectado, con fluorescentes de $70 \mathrm{~W}$.

- La programación de iluminación del anejo C se ha matizado con el estudio de iluminación natural realizado (Tabla 4), eliminando la iluminación artificial cuando se obtienen unos adecuados niveles de iluminación natural.

\subsection{Configuraciones}

La problemática de la eficiencia energética y el confort asociada a la iluminación natural y sus efectos térmicos se acrecienta en edificios con un alto porcentaje de acristalamiento, que buscan una mejora de los niveles de iluminación natural, ubicados en entornos con alta radiación solar, pudiendo aumentar la demanda energética del edificio (32). Por ello, el estudio presentado se centra en los meses más desfavorables de primavera y verano, siendo además el período de tiempo que inicialmente estaba previsto el uso del pabellón en cada una de las ubicaciones.

Para el estudio de las condiciones ambientales y energéticas del pabellón se parte de los modelos considerados, sobre los que se establecen diferentes variaciones que permitan comparar los resultados, para realizar una valoración de diferentes parámetros en las condiciones energéticas del pabellón en cada ubicación:

- Valoración de la contribución del lucernario en los niveles de iluminación natural, por lo que se analizan los distintos modelos considerando los cerramientos opacos de fachada.

- Valoración de la influencia del acristalamiento de la fachada. En Bruselas el pabellón presenta paramentos opacos junto con otros totalmente acristalados, por lo que se analiza esta configuración tal y como fue construida. Sin embargo, en Madrid existe una variación del porcentaje de acristalamiento, por lo que se analiza la variación del porcentaje de acristalamiento de la fachada a partir de los resultados más representativos obtenidos del estudio de iluminación natural en cada una de las orientaciones.

\section{ANÁLISIS Y VALORACIÓN DE LOS RESULTADOS}

\subsection{Valoración del lucernario}

La disposición de los lucernarios, considerando las fachadas opacas, proporciona un alto número de horas de soleamiento 
en el interior del pabellón en ambas ubicaciones. En la Figura 5 se puede observar cómo el número de horas de soleamiento en Madrid, con lucernarios en la orientación nortesur y este-oeste, es parecido al número de horas de Bruselas en el modelo B.1, con lucernario en la orientación este y oeste, mientras que en el modelo B.2 tiene la mitad de horas que el resto al tener sólo lucernario en orientación oeste.

En cuanto a la radiación solar, ésta es muy dispar en ambos modelos. Mientras que en Bruselas en los meses de verano no se alcanzan más de $500 \mathrm{~W} / \mathrm{m}^{2}$ en las fachadas este-oeste (Figura 6), en Madrid por el contrario existe un aumento de esta radiación, llegando a alcanzar los $850 \mathrm{~W} / \mathrm{m}^{2}$ en la fachada sur (Figura 7) y $800 \mathrm{~W} / \mathrm{m}^{2}$ en las este y oeste (Figura 8).

Si bien los valores FIN obtenidos son superiores al 1\% establecido por Baker y Steemers (33) para las distintas configuraciones (Tabla 5), el estudio dinámico de iluminación natural considerando unas condiciones de cielo despejado en verano, la época más desfavorable del año, refleja que la iluminación natural del pabellón en Bruselas presenta un peor comportamiento debido a los bajos niveles de radiación solar. Durante gran parte del día los niveles de iluminación del módulo central son bajos, no alcanzándose el valor de referencia de 300 lux (34), tal como demuestra el factor de Daylight Autonomy $\left(\mathrm{DA}_{300}\right)$ prácticamente nulo en el mes más favorable de junio, que indica que sólo ese porcentaje de horas al día se supera el nivel de referencia y con más de la mitad de la superficie del pabellón con valores de iluminación natural inferiores a 100 lux, debido a la insuficiente radiación solar en los meses de primavera y verano (Tabla 5).

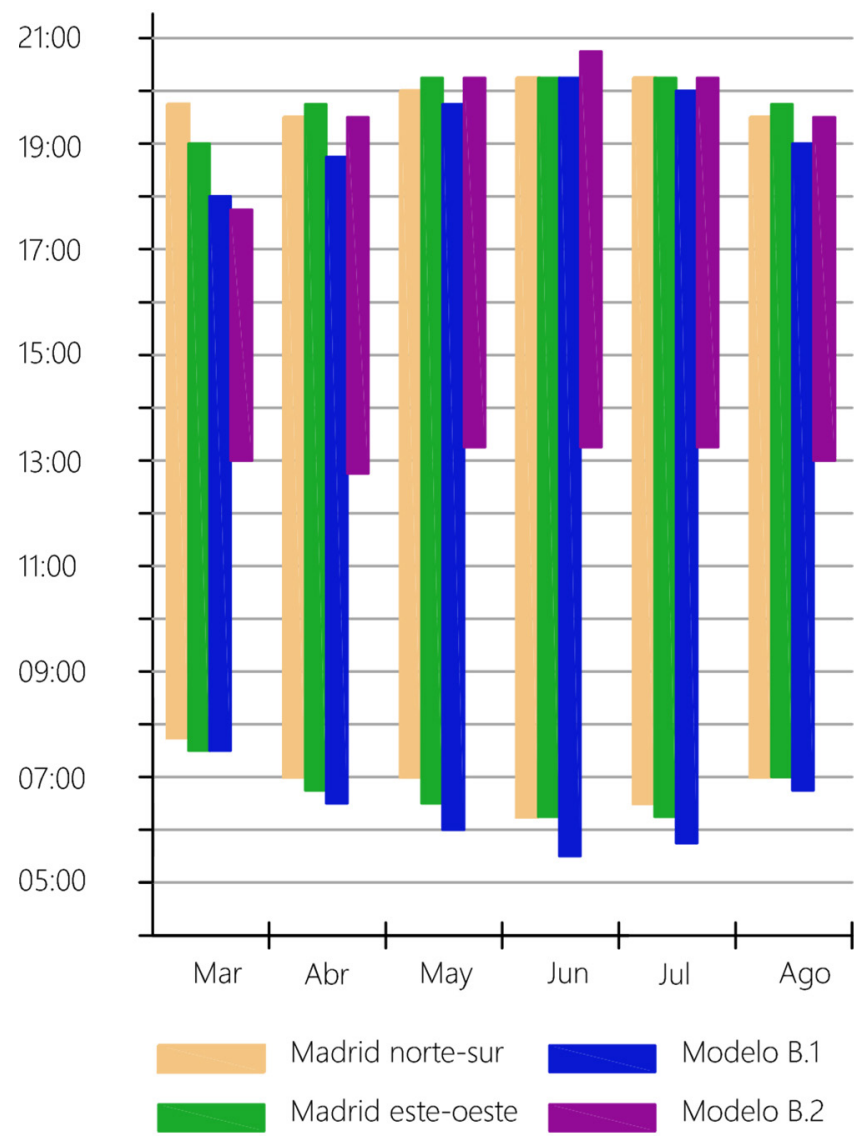

Figura 5. Número de horas de soleamiento en el interior del pabellón en Bruselas y Madrid.
En cambio en Madrid, tal como se puede apreciar en la Tabla 5, existe un mayor porcentaje de horas al día que superan el nivel de referencia en verano, siendo éste cercano al $50 \%$ debido a la menor profundidad del pabellón. Los porcentajes de iluminación inferiores a 100 lux, UDI ${ }_{<100}$, en Madrid son en torno al $20 \%$ mientras que en Bruselas ese porcentaje casi se triplica. En cuanto al UDI Ilega a $_{100}$ valores muy altos en Madrid, consecuente con los valores de $\mathrm{DA}_{300}$ que presenta; mientras que en Bruselas tiene valores bajos ya que en buena parte del día no se superan los 100 lux. En ningún caso se alcanzan niveles de deslumbramiento dentro del pabellón.

\subsection{Variación porcentaje de acristalamiento de fachadas}

Mientras que en Bruselas la configuración del pabellón presenta paramentos opacos con ladrillo cara vista o $100 \%$ acristalados, en Madrid el intento de adaptación del pabellón a un clima más severo queda referenciado en la diferente configuración de la envolvente vertical en función de la orientación, aumentando la superficie opaca de ladrillo, apareciendo un espacio más cerrado, iluminado por los huecos de fachada centrados en su mayoría en los patios interiores.

Así en Madrid en la orientación norte-sur aparecen fachadas acristaladas al $100 \%$, mientras que en la orientación este y oeste estos porcentajes varían desde el 40, 60 y $80 \%$. Esta variación del porcentaje de paramento acristalado nos permite conocer cómo influye este parámetro en los niveles de iluminación. Analizando los resultados del estudio de iluminación natural de los diferentes modelos de simulación que reproducen dichos porcentajes de variación del hueco en cada orientación, se observa que los resultados más representativos obtenidos corresponden a porcentajes de acristalamiento del 40, 60 y $100 \%$ en cada una de las orientaciones.

Los resultados de iluminación natural en Madrid mejoran al acristalar los paramentos de las fachadas (Tabla 6), llegando a existir en verano zonas con deslumbramiento en el interior del pabellón cuando las fachadas cuentan con un $100 \%$ de vidrio en ellas, al igual que en Bruselas, aunque con unos niveles inferiores.

En la evolución del comportamiento energético se ha analizado la evolución horaria de la temperatura operativa interior en libre evolución, que se compara con la banda de confort, junto con los niveles globales de radiación solar, lo que permite relacionar la evolución de la temperatura con el soleamiento e iluminación del pabellón. Este análisis se realiza para tasas de ventilación de 0,5, 1 y 2 renovaciones/hora para conocer la influencia de este parámetro.

Térmicamente el comportamiento del pabellón en Bruselas es mejor que en Madrid, principalmente en verano, con temperaturas más moderadas y cercanas a la banda de confort, llegando en ocasiones a estar dentro de la misma (Figura 6). En Madrid, tanto en los meses templados como los calurosos, hay una mayor oscilación térmica interior, de 12 a $25{ }^{\circ} \mathrm{C}$ (Figura 7). La temperatura operativa interior máxima se produce tras alcanzarse los valores máximos de radiación solar, coincidiendo con los valores máximos de temperatura exterior, aumentando con el porcentaje de acristalamiento, debido a la escasa inercia térmica del edificio. 

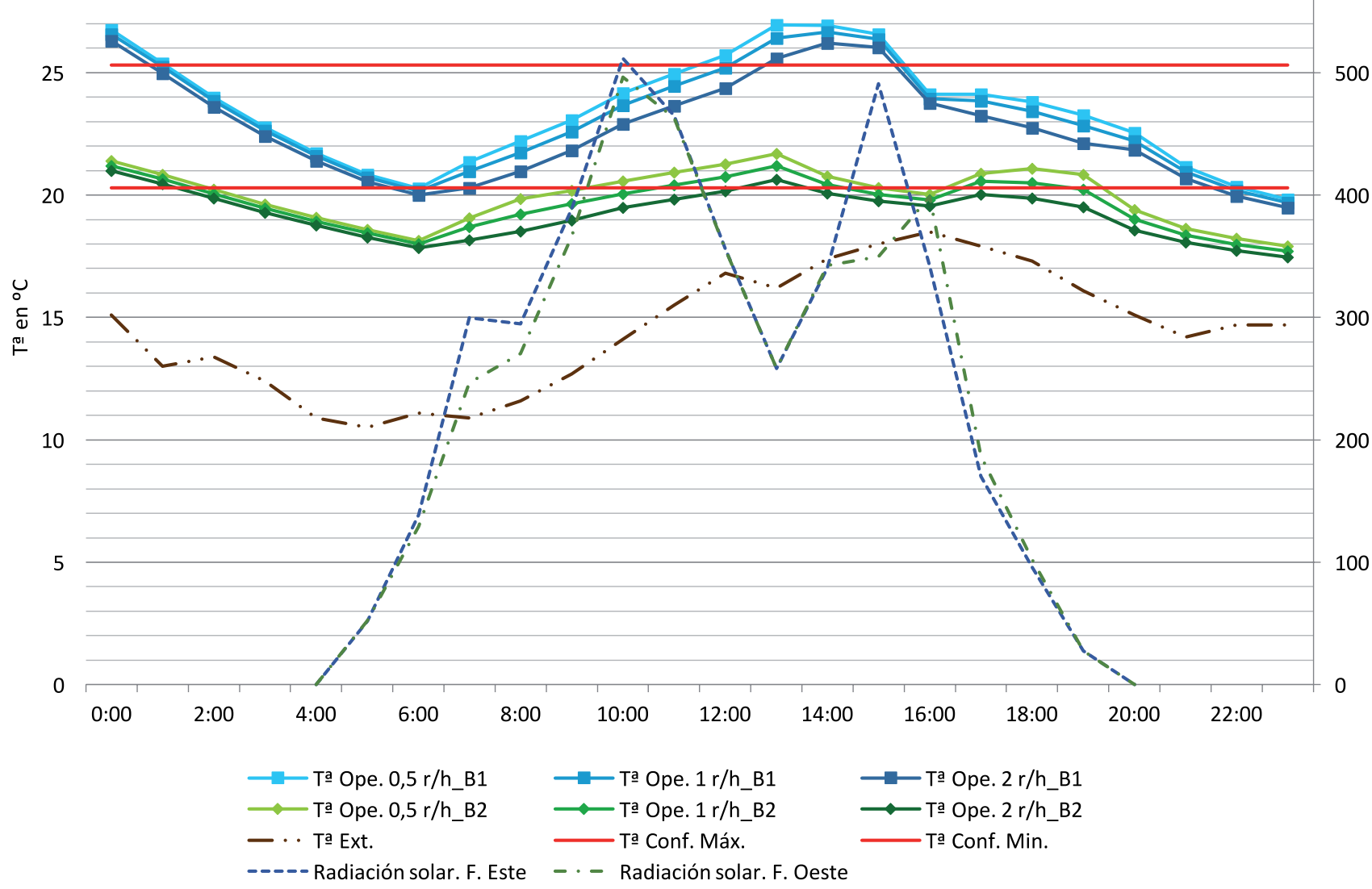

25

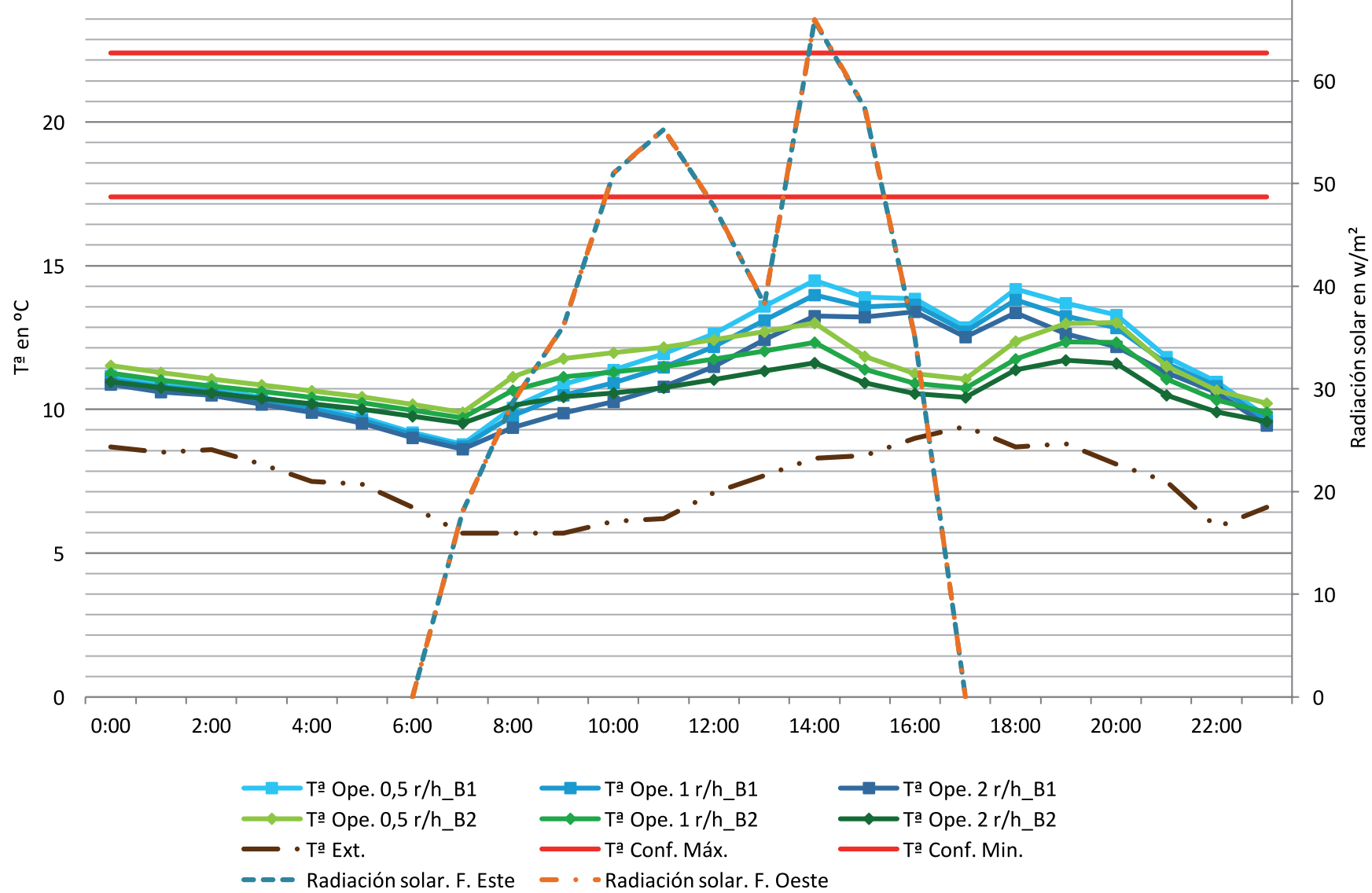

Figura 6. Evolución horaria de la temperatura operativa interior, temperatura exterior y radiación solar en Bruselas: 21 de junio (superior), 21 de marzo (inferior). 

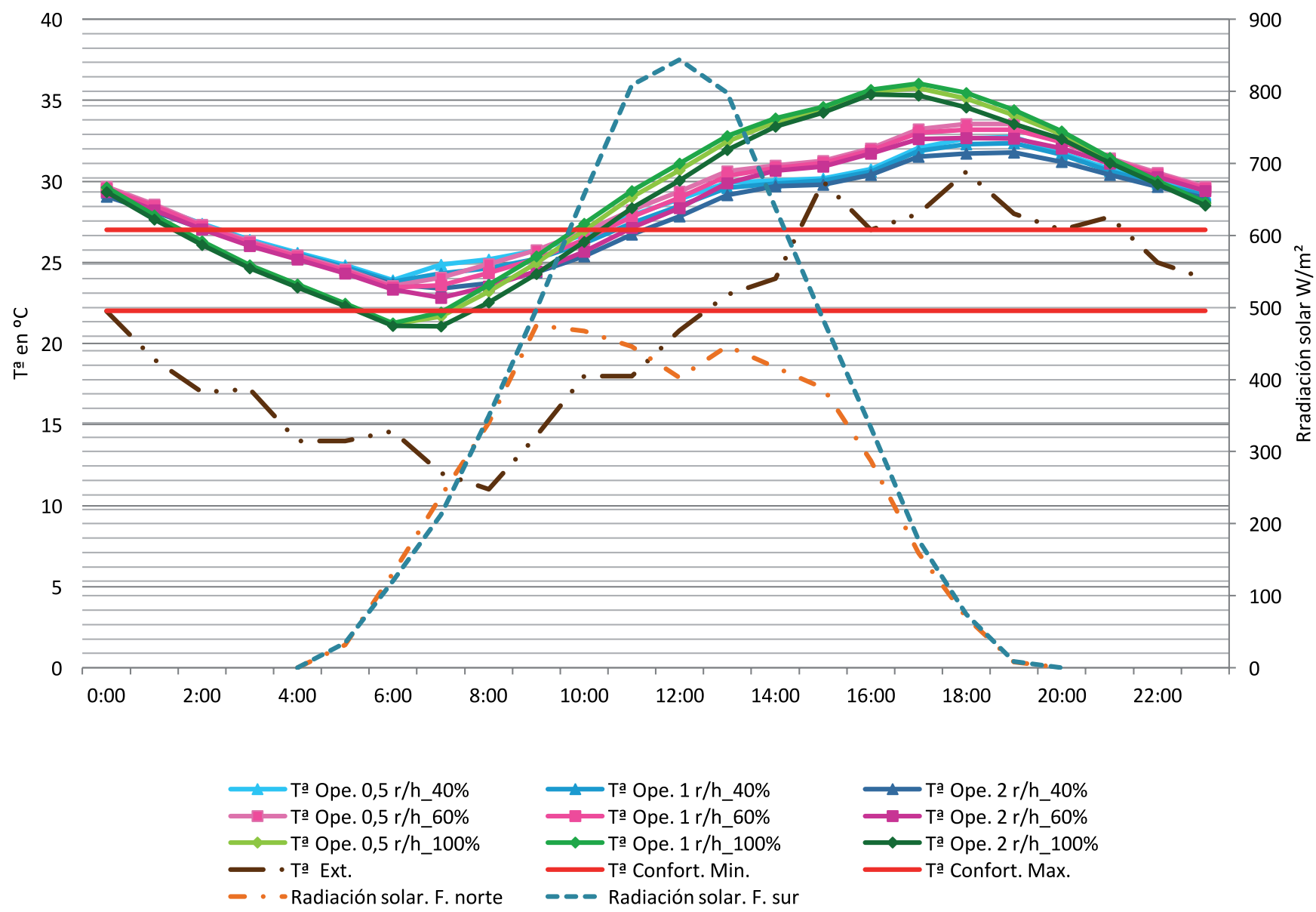

45

- Radiación solar. F. norte

- Radiación solar. F. sur
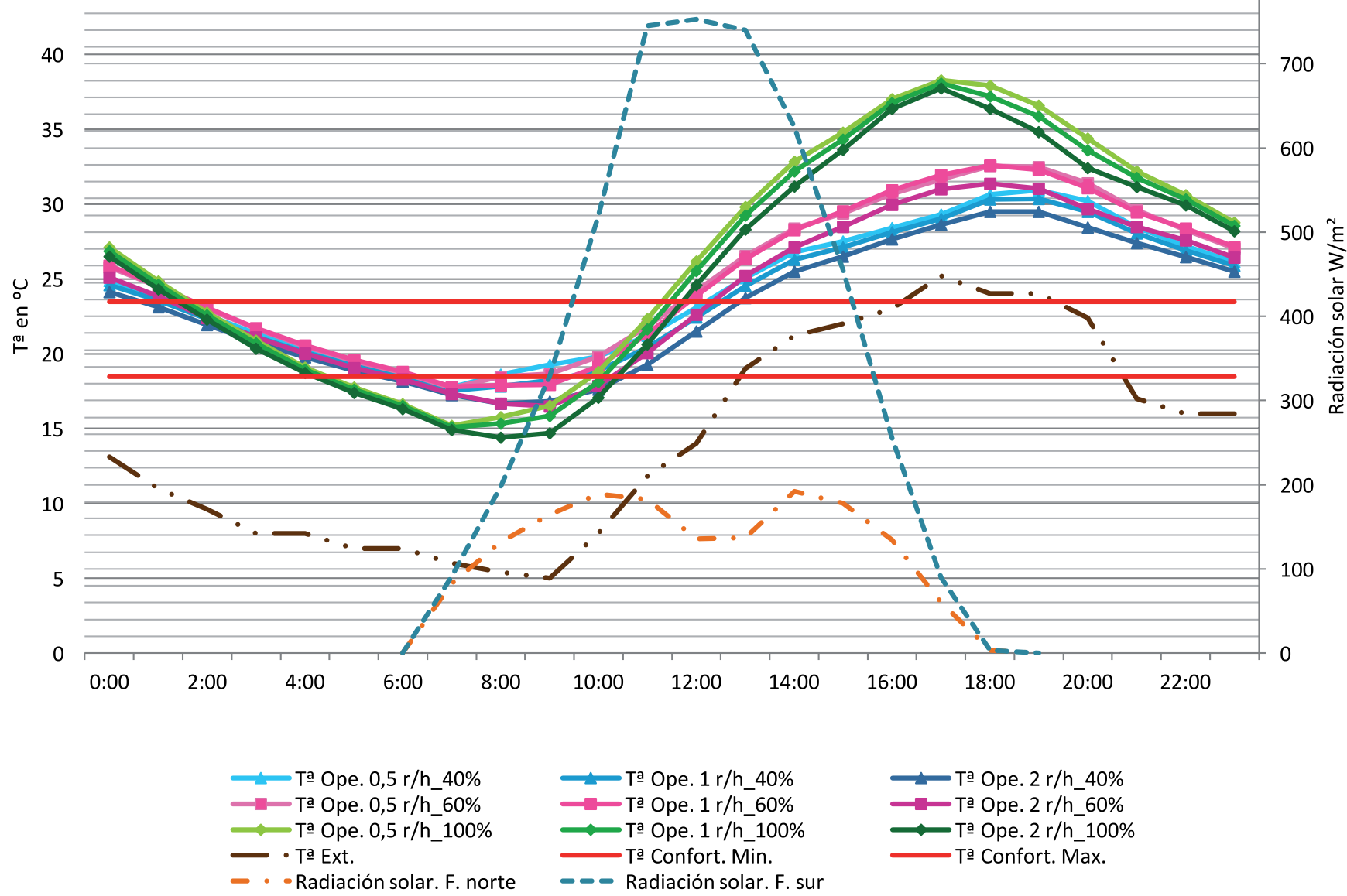

Figura 7. Evolución horaria de la temperatura operativa interior, temperatura exterior y radiación solar en Madrid, orientación sur: 21 de junio (superior), 21 de marzo (inferior). 

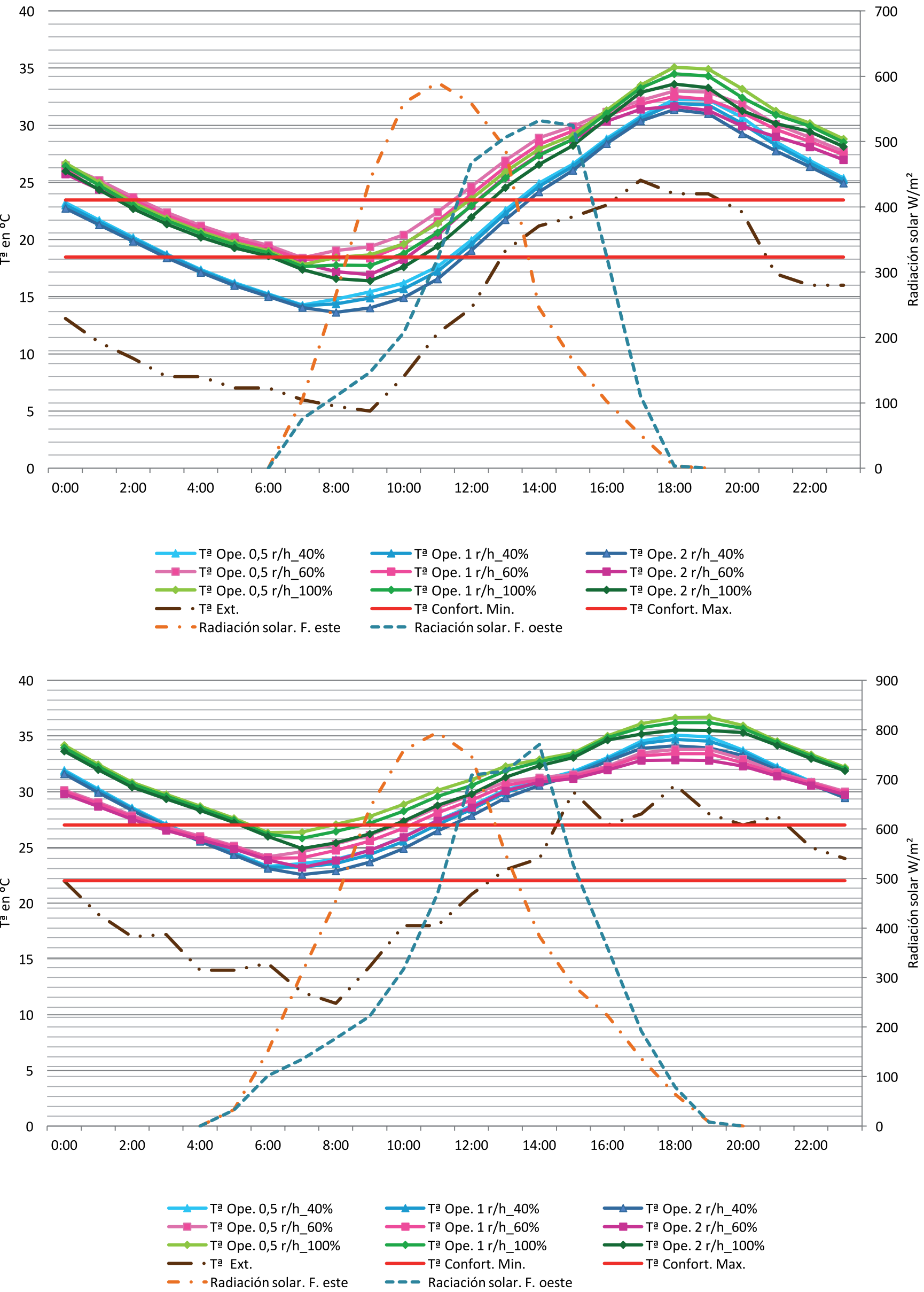

Figura 8. Evolución horaria de la temperatura operativa interior, temperatura exterior y radiación solar en Madrid, orientación oeste: 21 de junio (superior), 21 de marzo (inferior) 
Tabla 5. Valores de parámetros de iluminación natural debido a la acción de los lucernarios considerando las fachadas opacas. Mes de junio.

\begin{tabular}{|l|c|c|c|c|}
\hline \multirow{2}{*}{} & \multicolumn{2}{|c|}{ Bruselas } & \multicolumn{2}{c|}{ Madrid } \\
\cline { 2 - 5 } & Modelo B.1 & Modelo B.2 & \multicolumn{2}{c|}{ Modelo M } \\
\hline Orientación lucernario & Este-oeste & Oeste & Norte-sur & $1,8 \%$ \\
\hline FIN & $1,7 \%$ & $1,3 \%$ & $49,0 \%$ & $51,7 \%$ \\
\hline DA $_{300}$ & $0,0 \%$ & $2,1 \%$ & $19,9 \%$ & $19,7 \%$ \\
\hline UDI $_{<100 \text { lux }}$ & $60,6 \%$ & $71,5 \%$ & $80,1 \%$ & $80,3 \%$ \\
\hline UDI $_{100-2.000 \text { lux }}$ & $39,4 \%$ & $28,5 \%$ & $0,0 \%$ & $0,0 \%$ \\
\hline UDI $_{>2.000 \text { lux }}$ & $0,0 \%$ & $0,0 \%$ & & 0 . $\%$ \\
\hline
\end{tabular}

Tabla 6. Valores de parámetros de iluminación natural en función del acristalamiento de la fachada. Mes de junio.

\begin{tabular}{|c|c|c|c|c|c|c|c|c|}
\hline \multirow{4}{*}{\begin{tabular}{|l|} 
\\
Orientación lucernario \\
\% acristalamiento \\
\end{tabular}} & \multicolumn{2}{|c|}{ Bruselas } & \multirow{2}{*}{\multicolumn{6}{|c|}{$\begin{array}{c}\text { Madrid } \\
\text { Modelo M }\end{array}$}} \\
\hline & \multirow{3}{*}{$\begin{array}{c}\text { Modelo B.1 } \\
\text { Este-oeste } \\
100 \text { E o O } \\
\end{array}$} & \multirow{3}{*}{\begin{tabular}{|c|} 
Modelo B.2 \\
Oeste \\
50 \\
\end{tabular}} & & & & & & \\
\hline & & & & Norte-su & & & iste-oest & \\
\hline & & & 100 & 60 & 40 & 100 & 60 & 40 \\
\hline FIN & $11,4 \%$ & $6,1 \%$ & $8,9 \%$ & $6,6 \%$ & $5,3 \%$ & $8,9 \%$ & $6,6 \%$ & $5,3 \%$ \\
\hline $\mathrm{DA}_{300}$ & $74,2 \%$ & $46,49 \%$ & $75,5 \%$ & $72,9 \%$ & $70,3 \%$ & $84,6 \%$ & $82,8 \%$ & $73,4 \%$ \\
\hline $\mathrm{UDI}_{<100 \text { lux }}$ & $12,7 \%$ & $21,7 \%$ & $11,0 \%$ & $13,4 \%$ & $13,5 \%$ & $5,6 \%$ & $6,8 \%$ & $7,8 \%$ \\
\hline UDI $_{\text {100-2.000 lux }}$ & $84,3 \%$ & $76,9 \%$ & $82,6 \%$ & $86,5 \%$ & $86,4 \%$ & $88,7 \%$ & $99,7 \%$ & $92,2 \%$ \\
\hline $\mathrm{UDI}_{>2.000 \text { lux }}$ & $3,0 \%$ & $1,3 \%$ & $6,4 \%$ & $0,0 \%$ & $0,0 \%$ & $5,7 \%$ & $0,0 \%$ & $0,0 \%$ \\
\hline
\end{tabular}

Durante el verano en Madrid, en el mes de junio, en las horas centrales del día existen niveles de iluminación natural medios que superan el nivel de referencia de 300 lux en las orientaciones principales y bajo cielo nublado y despejado, llegando a alcanzar valores mínimos en orientación sur de 500 lux y máximos de 1.400 lux en orientación este y oeste cuando la radiación solar tiene un valor máximo de $800 \mathrm{~W} / \mathrm{m}^{2}$. La importante radiación solar, que conlleva altos niveles de iluminación interior, junto con las elevadas temperaturas exteriores provoca que por la tarde se puedan alcanzar temperaturas máximas de $36^{\circ} \mathrm{C}$, muy alejadas de las condiciones de confort (Figura 7).

En el mes de marzo durante las horas centrales del día, cuando la radiación solar es máxima con $800 \mathrm{~W} / \mathrm{m}^{2}$, los valores de iluminación natural medios oscilan entre 350-650 lux en orientación sur y entre 350 y 1400 lux en orientación este y oeste para condiciones de cielo nublado, aumentando estos valores en cielo despejado a 800 y 1.400 lux en orientación sur y disminuyendo en este y oeste a 600 y 1.000 lux. Al igual que sucede en verano la radiación solar y temperatura exterior son responsables de valores máximos de temperatura operativa interior (Figura 8).

\section{CONCLUSIONES}

La caracterización y valoración lumínica, ambiental y energética de las dos etapas temporales del pabellón de España de Corrales y Molezún, en Bruselas y Madrid, permiten realizar una valoración de su módulo espacial como elemento de acondicionamiento ambiental.

El pabellón de España, en su primera localización en Bruselas, presenta un adecuado comportamiento para el período en el que estuvo abierto al público, primavera y verano; con una adecuada conducta en cuanto a valores energéticos se refiere, presentando una temperatura operativa interior con valores cercanos o dentro de la banda de confort en verano. Sin em- bargo, los niveles de iluminación natural no alcanzan en gran parte del tiempo el nivel mínimo de referencia de 300 lux, establecidos para edificios con uso expositivo. Por el contrario, tras ser trasladado a Madrid se modifica su configuración formal, presentando el pabellón un comportamiento ambiental totalmente distinto, con una notable mejora de los niveles de iluminación natural, debido al aumento de la radiación solar, pero con una evolución de la temperatura operativa interior alejada de las condiciones de confort. Este contraste se debe a la influencia de varios factores:

- El clima. En Bruselas era suave, con temperaturas exteriores máximas de $23^{\circ} \mathrm{C}$ en verano; mientras que en Madrid el clima es más cálido alcanzándose temperaturas superiores a $30^{\circ} \mathrm{C}$ en verano.

- La compacidad. En Bruselas el pabellón es un elemento alargado y profundo, compacto, con un valor de compacidad de 9,10, mientras que en Madrid el pabellón se convierte en un elemento sin profundidad, con patios interiores, aumentando en casi el doble la superficie de fachada, disminuyendo el valor de compacidad a 3,85 , lo que supone un incremento de los intercambios energéticos.

- La orientación. En Bruselas el pabellón tiene los cerramientos verticales con dos orientaciones, la mitad orientada en norte-sur y la otra mitad en este-oeste, por el contrario los huecos de los lucernarios de todo el pabellón estaban orientados en este-oeste, lo que proporcionaba una mejora de los niveles de radiación en esos huecos durante el verano. En cambio, en Madrid los cerramientos tienen tanto orientación norte-sur como este-oeste, al igual que los lucernarios, recibiendo mayor radiación solar y existiendo unos valores energéticos y de iluminación natural menos estables.

El pabellón de España se caracteriza por el uso de un módulo hexagonal autónomo, tanto en su estructura como con la iluminación gracias al lucernario que ilumina el interior del edificio. Sin embargo este lucernario es insuficiente para garantizar los niveles de iluminación natural en el interior del 
pabellón, por lo que se debe complementar con iluminación natural a través de los huecos de fachada.

Una de las principales deficiencias del pabellón de España es la materialidad de la envolvente, de baja calidad térmica y escasa inercia térmica, que conlleva que el pabellón intercambie calor rápidamente con el exterior, proceso que en Madrid se acentúa al aumentar el porcentaje de acristalamiento, lo que se traduce en una falta de homogeneidad y una importante oscilación térmica, impidiendo el control de la evolución de la temperatura operativa interior debido a la radiación solar.

Para una adecuada respuesta ambiental de este tipo de arquitectura modular, entendida como sistemas reutilizables en múltiples emplazamientos, se deberían tener en cuenta varios condicionantes en relación con su respuesta ambiental:
- Las operaciones de adición de los módulos deberían atender a una forma lo más compacta posible, disminuyendo en lo posible la superficie de envolvente.

- El perímetro se convierte en el elemento de protección térmica, independientemente de la versatilidad de los sistemas de crecimiento y profundidad de la planta, así como de refuerzo de la iluminación natural. Dada su generalmente escasa inercia térmica deberían incorporarse unos adecuados niveles de aislamiento térmico en la envolvente opaca, tanto de fachada como de cubierta.

- En función de la zona climática y la orientación debería evaluarse un adecuado porcentaje de superficie acristalada que garantice unos apropiados niveles de iluminación natural, sin que los efectos de una alta radiación solar pueda generar problemas de confort térmico, junto con una mejora de la transmitancia térmica de los huecos acristalados.

\section{REFERENCIAS}

(1) Risselada, M., et al. (2011). Alison \& Peter Smithson: a critical anthology, Barcelona: Polígrafa.

(2) Cassinello, P. (2008). Eduardo Torroja y la industrialización de la "machine à habiter" 1949-1961. Informes de la Construcción, 60(512): 5-18, doi: http://dx.doi.org/10.3989/ic.08.031.

(3) Gideon, S. (1978). La mecanización toma el mando, Barcelona: Gustavo Gili.

(4) Tafuri, M. (1997). Teoría e historia de la arquitectura, Barcelona: Laia.

(5) Olgyay, V. (1963). Design with Climate: Bioclimatic Approach to Architectural Regionalism, Princeton: Princeton University Press.

(6) Banham, R. (1969). The architecture of the well-tempered environment, London: Architectural Press.

(7) Porteous, C. (2002). The New eco-Architecture: Alternatives from the Modern Movement, London: Spon Press.

(8) Hawkes, D. (2012). Architecture and Climate: An Environmental History of British Architecture, 1600 - 200o, New York: Routledge.

(9) Cole, R. J. (2012). Accommodating the British climate. Building Research \& Information, 40(5): 638-642, doi: http:// dx.doi.org/10.1080/09613218.2012.711992.

(10) Wadel, G., Avellaneda, J., Cuchí, A. (2010). La sostenibilidad en la arquitectura industrializada: cerrando el ciclo de los materiales. Informes de la Construcción, 62(517): 37-51, doi: http://dx.doi.org/10.3989/ic.09.067.

(11) Requena-Ruiz, I. (2012). Bioclimatism in the Architecture of Le Corbusier: The Millowners Association Building. Informes de la Construcción, 64 (528): 549-562, doi: http://dx.doi.org/10.3989/ic.11.121.

(12) Ramírez, C., Nieto, E., Narbona, G., Sendra, J. J., Suárez, R. (2015). Numerical simulation of the temperature evolution in a room with a mur neutralisant. Application to "The City of Refuge" by Le Corbusier. Energy \& Buildings, 86: 708722, doi: http://dx.doi.org/10.1016/j.enbuild.2014.10.032.

(13) Escandón, R., Suárez, R., Sendra, J. J.(2015). Energy and climate simulation in the Upper Lawn Pavilion, an experimental laboratory in the architecture of the Smithsons. Building Simulation. 8(1): 99-109, doi: http://dx.doi.org/10.1007/ s12273-014-0197-0.

(14) Moneo, R. (1993). Optimismo racionalista. Arquitectura Viva, 28: 72-73.

(15) AA.VV. (2004). Corrales y Molezún. Pabellón de España en la Exposición Universal de Bruselas 1958, Madrid 1959, p. 25, Arquitecturas ausentes del siglo XX. Madrid.

(16) Cánovas, A. (Eds.) (2005). Pabellón de Bruselas '58: Corrales y Molezún. Madrid: Ministerio de la Vivienda.

(17) Fernández del Amo, J. L. (1969). Nuevo Pabellón del Ministerio de Agricultura en la Feria Internacional del Campo, Madrid. Arquitectura, 121: 61.

(18) Corrales, J. A., Molezún, R. (2005). Memoria general. En Cánovas, A. (Eds.) Pabellón de Bruselas '58: Corrales y Molezún. Madrid: Ministerio de la Vivienda.

(19) Clarke, J., Janak, M. (1998). Simulating the thermal effects of daylight controlled lighting. Building Performance (BE$P A C U K)$, Issue 1.

(20) Franzetti, C., Fraisse, G., Achard, G. (2004). Influence of the coupling between daylight and artificial lighting on thermal loads in office buildings. Energy and Buildings 36(2): 117-126, doi: http://dx.doi.org/10.1016/j.enbuild.2003.10.005.

(21) Hviid, C. A., Nielsen, T. R., Svendsen, S. (2008). Simple tool to evaluate the impact of daylight on building energy consumption. Solar Energy, 82(9): 787-798.

(22) Autodesk Ecotect Analysis [versión 5.6]. https://www.autodesk.com.

(23) Daylight Visualizer. http://viz.velux.com/.

(24) Acosta, I., Muñoz, M., Esquivias, P., Moreno, D., Navarro, J. (2015). Analysis of the accurancy of sky component calculation in daylighting simulation programs. Solar Energy, 119: 54-67, doi: http://dx.doi.org/10.1016/j.solener.2015.06.022.

(25) DesignBuilder [versión 2.3.6.005]. https://www.designbuilder.co.uk/.

(26) Moon, P., Spencer, D. E. (1942). Illumination form a non-uniform sky. Illum. Eng., 37: 707-726. 
(27) Reinhart, C. F., Mardaljevic, J., Rogers, Z. (2006). Dynamic Daylight Performance Metrics for Sustainable Building Design. LEUKOS, 3(1): 7-31.

(28) Reinhart, C. F., Wienold, J. (2011). The day lighting dashboard. A simulation-based design analysis for daylit spaces. Building and Environment, 46: 386-396, doi: http://dx.doi.org/10.1016/j.buildenv.2010.08.001.

(29) Reinhart, C., Walkenhorst, O. (2001). Validation of dynamic RADIANCE-based daylight simulations for a test office with external blinds. Energy \& Buildings, 33: 683-697, doi: http://dx.doi.org/10.1016/so378-7788(01)ooo58-5.

(30) Nabil, A., Mardaljevic, J. (2005). Useful Daylight Illuminance: A New Paradigm to Access Daylight in Buildings. Lighting Research Technology, 37: 41-59.

(31) De Dear, R. J., Brager, G. S. (1997). ASHRAE RP-884 Final Report: developing an adaptive model of thermal comfort and preference. Atlanta: American Society of Heating, Refrigerating and Air-Conditioning Engineers.

(32) Tzempelikos, A., Athienitis, A. K. (2007). The impact of shading design and control on building cooling and lighting demand. Solar Energy, 81(3): 369-382, doi: http://dx.doi.org/10.1016/j.solener.2006.06.015.

(33) Baker, N., Steemers, K. (2002). Daylight. Design of Buildings. London: James\&James.

(34) AENOR (2012). UNE-EN 12464-1:2012 Iluminación. Iluminación de los lugares de trabajo. Parte 1: Lugares de trabajo en interiores. Asociación Española de Normalización (AENOR). 